\title{
9
}

\section{Semi-Centralized Routing Algorithms for 3-Stage Clos Networks}

\author{
F.K. Liotopoulos and S. Chalasani ${ }^{\mathrm{a} *}$ \\ ${ }^{a}$ Dept. of Electrical and Computer Engineering, \\ University of Wisconsin-Madison, Madison, WI 53706-1691 \\ e-mail: $\{$ fotios, suresh\}oece.wisc.edu
}

In this paper we study the switching performance of three-stage Clos networks. First, we consider an existing control algorithm for operating the asymmetrical Clos networks in the nonblocking mode, and we derive sufficient conditions under which these networks are nonblocking for this control algorithm. We next design a new routing algorithm for nonblocking operation of asymmetrical Clos networks. Using simulation results, the new control algorithm is shown to perform better than the previous one in terms of network utilization, blocking probability and fault-tolerance. In order to further study the performance of the new semi-centralized routing algorithm, we introduce a queuing model and solve it using an exact mean value analysis algorithm. Simulation results are used to verify the predictions of our analysis regarding the router's throughput, latency, and the network's capacity utilization. Since semi-centralized routers are performancelimited by their centralized bottlenecks, we introduce a routing scheme, which consists of multiple interdependent routers which operate in parallel. Simulation results indicate that this routing scheme substantially improves the performance of the single router, thus alleviating the congestion effects due to the centralized bottlenecks.

Keyword Codes: I.6.4; I.6.5; I.6.6; C.2.1; C.2.3; C.2.4

Keywords: Model Validation and Analysis; Model Development; Simulation Output Analysis; Network Architecture and Design; Network Operations; Distributed Systems

\section{INTRODUCTION}

Three-stage Clos networks [4] have been used for multiprocessor interconnection as well as for data communication. For example, the Memphis switch of the IBM GF-11 parallel computer uses a symmetric three-stage Clos network to interconnect 576 processors [1]. Due to their low blocking probability, three-stage Clos networks have also been advocated for ATM (Asynchronous Transfer Mode) networks [5]. A variant of the three-stage Clos network has been designed for use in the High-Performance Switching System project at the Lawrence Livermore National Laboratory [22].

A three-stage Clos network consists of three successive stages of switching elements which are interconnected by links. In a symmetric three-stage network, all switching

\footnotetext{
*This research was supported in part by a grant from the Graduate School of the University of WisconsinMadison and the NSF grants CCR-9308966 and ECS-9216308. Fotios Liotopoulos was supported by a Vilas Graduate Fellowship from the University of Wisconsin-Madison. A part of the results presented in this paper has appeared in the Proceedings of the 23rd International Conference on Parallel Processing, vol.I, pp.101-108, 1994.
} 
elements in a stage are uniform (see Figure 1). In the symmetric Clos network of Figure 1, there are $r$ switches of size $n \times m$ in the first stage, $m$ switches of size $r \times r$ in the second, and $r$ switches of size $m \times n$ in the third. This network thus interconnects $n \cdot r$ input ports with $n \cdot r$ output ports.

Three-stage Clos networks can be designed to operate either in the rearrangeable mode or in the nonblocking mode. In a rearrangeable network, a connection between any idle input port and any idle output port can always be realized by rearranging some of the existing connections if necessary. In a nonblocking network, an idle pair of ports can be connected without rearranging existing connections. Due to their more stringent requirements, nonblocking networks, in general, require more hardware resources than rearrangeable networks. For example, it has been shown that the symmetric three-stage Clos network of Figure 1 is rearrangeable if $m \geq n$, while the same network is nonblocking if $m \geq 2 n-1$ [2]. Several other researchers considered symmetric three-stage Clos networks in the context of either rearrangeable or nonblocking operation $[6,12,18,8]$.

Masson and Jordan introduced asymmetrical three-stage Clos networks as a generalization of the symmetric Clos network of Figure 1 [16]. These networks have $r_{1}$ switches of size $n_{1} \times m$ in the first stage and $r_{2}$ switches of size $m \times n_{2}$ in the third; there are $m$ switches in the middle stage each of size $r_{1} \times r_{2}$. Hwang proved many results on the rearrangeability of these networks in a simple manner [7]. Hwang and Jajszczyk developed many routing strategies for these asymmetrical three-stage networks, and derived conditions under which these routing strategies enable nonblocking operation of the networks [9]. Koppelman considered more general asymmetrical Clos networks than those considered by Masson and Jordan, and Hwang and Jajszczyk. The networks considered by Koppelman may have nonuniform switches in each stage and arbitrary connection patterns between stages; he studied the rearrangeability of these networks [13]. Varma and Chalasani also designed connection-assignment algorithms for asymmetrical Clos networks under the rearrangeable mode of operation [21].

Ohta considered a symmetric three-stage Clos network, similar to the one shown in Figure 1, and derived conditions under which it is nearly-nonblocking [17]. Similar to a nonblocking network, a nearly-nonblocking network does not allow any rearrangement of the existing connections in order to establish a new connection; however, unlike a nonblocking switch, a nearly-nonblocking switch allows at most one rearrangement to take place when an existing connection is disconnected. Thus, the overhead of realizing a new connection under the nearly-nonblocking mode of operation is the same as that of in the nonblocking operation. However, the overhead of disconnection is in the nearlynonblocking mode of operation is slightly more than that in the nonblocking operation. This increase in overhead is justified, since the hardware resource required for nearlynonblocking operation are much lower than those required for nonblocking operation [17]. In other words, for a given network, nearly-nonblocking operation utilizes it better than the nonblocking mode of operation.

In this paper, we consider a general class of asymmetrical three-stage Clos networks [21] and study their nearly-nonblocking operation. The motivation to study asymmetrical Clos networks is two-fold. First, asymmetrical Clos networks permit the use of heterogenous components, which may be the natural choices for building a distributed network. Second, even a symmetric network may be rendered asymmetrical due to link and switch failures; 


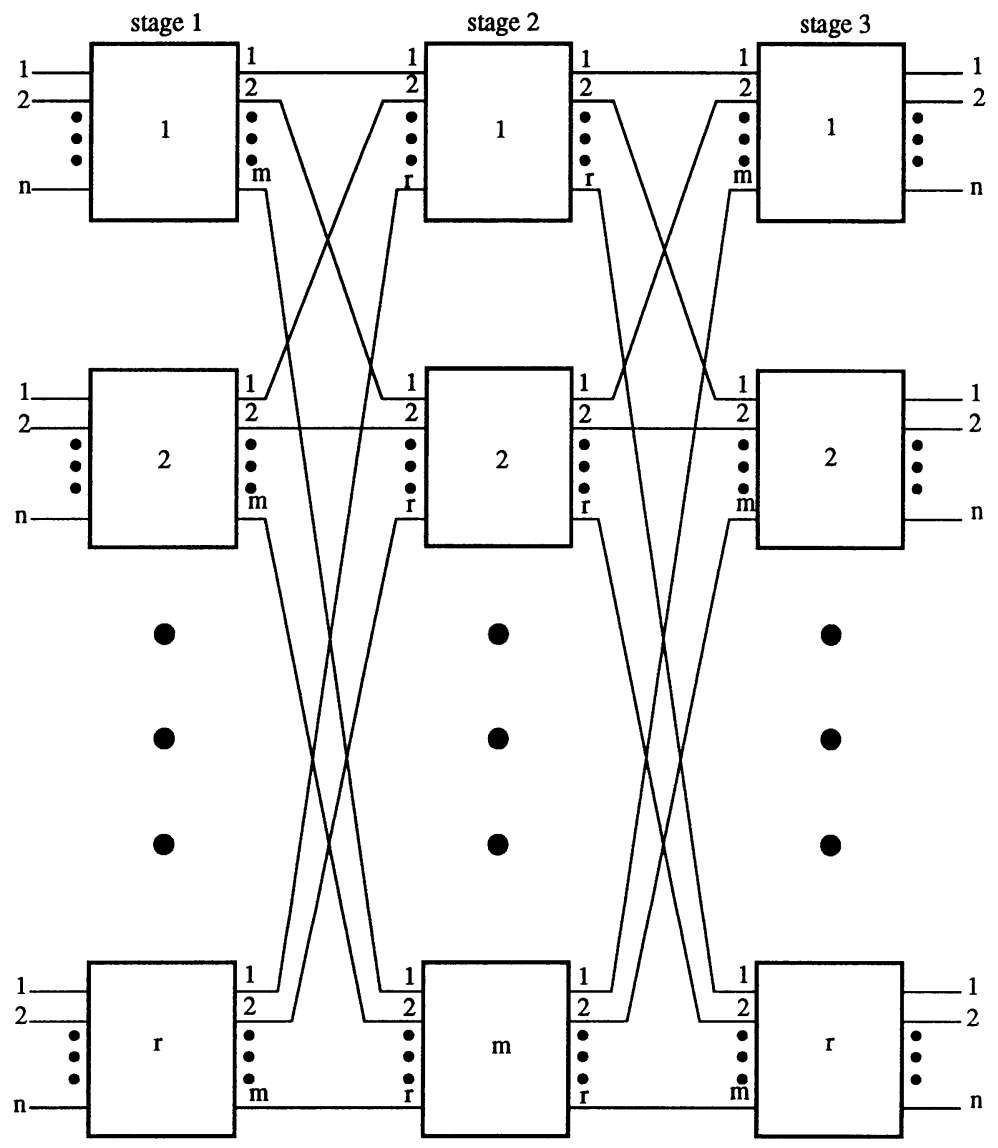

Figure 1. A symmetric three-stage Clos network. 
the results reported in this paper allow a symmetric network to operate in the nearlynonblocking mode despite the presence of network faults.

Given a connection request from an input port to an output port of a network switch, a routing decision has to be made in order for a path to be established. This decision should be fast and should efficiently allocate the network's resources (i.e., links, buffers and switches), in order to achieve high capacity and bandwidth utilization as well as low blocking probability (for blocking switches).

Decentralized (or fully distributed) routing is attractive because it reduces the latency of the routing decision, by exploiting the parallelism that exists in it. Distributed routing solutions scale efficiently with the network's capacity and they usually do not create bottlenecks for uniform traffic.

Their main disadvantage is that the decisions are made by considering only local information. Therefore, they are usually less efficient in controlling the overall network utilization and load distribution.

Communication of global information about the state of the network sometimes results in higher network traffic, longer routing latencies and reduced performance-scalability of routing algorithms. But, on the other hand, more "intelligent" decisions can be made, thus achieving more efficient traffic control over the network. Such a (semi)-centralized approach is the subject of our study in this paper.

In order to study the effect of the centralized bottleneck that exists in (semi)-centralized, circuit switching routers, we develop a queuing model and solve it with an exact Mean Value Analysis (MVA) method, which predicts the performance of such a router.

The work reported in $[13,21,17]$ is most relevant to the work reported in this paper. The results reported in this paper are applicable for the general asymmetrical Clos networks described in [13, 21]; however, while Koppelman and Varma and Chalasani consider rearrangeable operation of these networks, we consider nearly-nonblocking operation of these networks. Ohta developed a distributed control algorithm for nearly-nonblocking operation of symmetric Clos networks [17]. Our results differ from the results of Ohta [17] in that we develop a semi-centralized control algorithm for nearly-nonblocking operation of asymmetrical Clos networks. We also present a queuing model to predict the performance of the router.

The rest of this paper is organized as follows. Section 2 introduces the general class of asymmetrical Clos networks studied in this paper. Sections 3 and 4 briefly discuss Ohta's distributed routing algorithm and describe in more detail our semi-centralized algorithm for nearly-nonblocking operation of asymmetrical Clos networks. Section 5 presents a performance evaluation of the proposed control algorithm described in the previous sections. Based on simulation results, we show that our semi-centralized algorithm can achieve better performance, compared to a fully distributed approach. In Section 6, we introduce a model for the router and the mean value analysis to predict its performance. Section 7 validates this model. Finally, Section 8 concludes this paper with an overview of our contributions and directions for future work. 


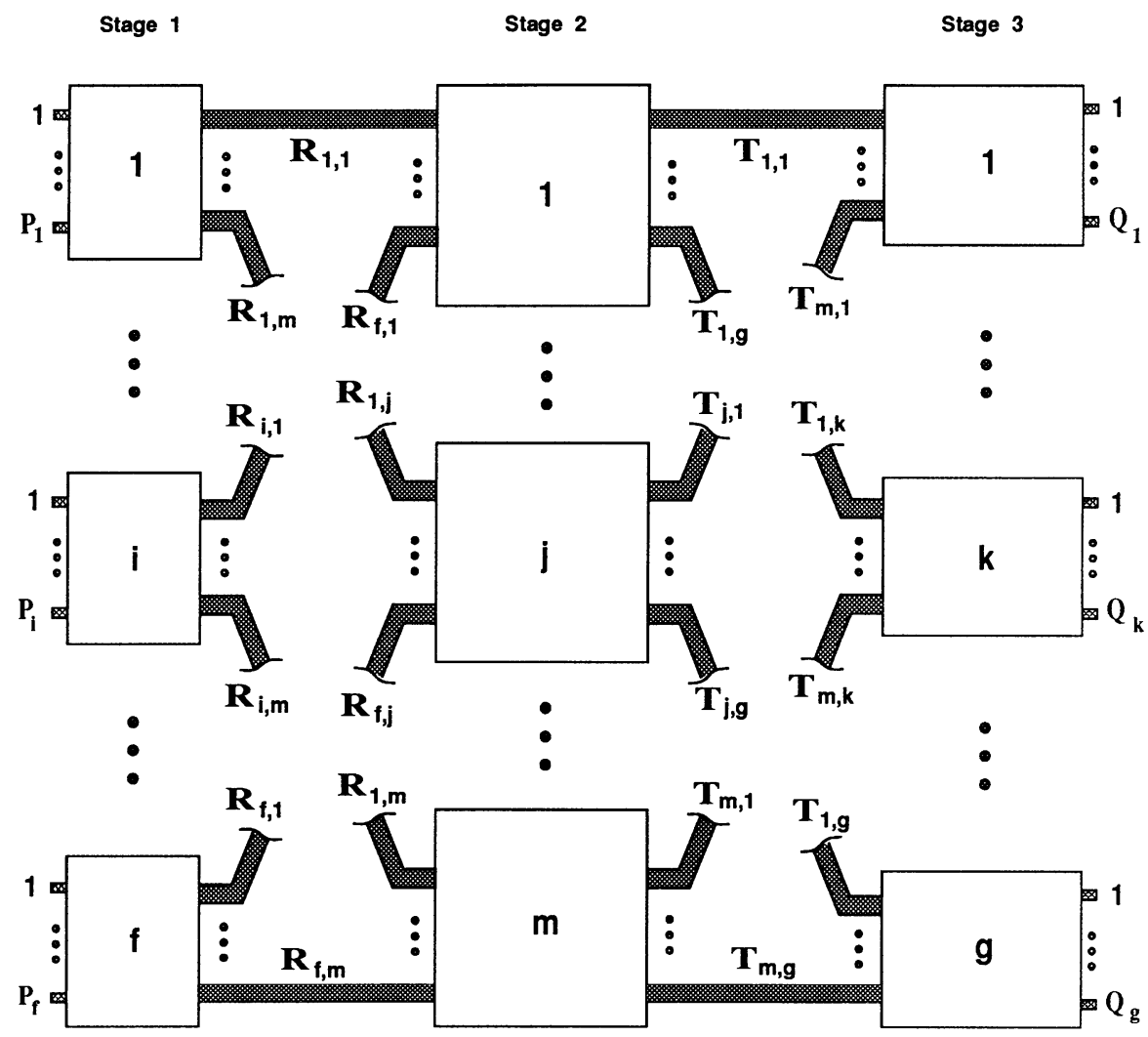

Figure 2. A general asymmetrical Clos network.

\section{PRELIMINARIES}

Figure 2 illustrates the general class of asymmetrical three-stage Clos networks considered in this paper. The set of switching elements in stage $i$ of this network will be denoted by $S_{i}$, for $i=1,2,3$. The number of switching elements in the three stages are $f, m$, and $g$, respectively. The interconnection pattern among the three stages is described below.

Stage 1 Switching element $i$ has $P_{i}$ input ports. It is connected to switching element $j$ in the middle stage via a set of $R_{i, j}$ links.

Stage 3 Switching element $k$ has $Q_{k}$ output ports. It is connected to switching element $j$ in the middle stage via a set of $T_{j, k}$ links.

The total number of input ports in the network (i.e. inputs to the first stage) is given by $\mathcal{I} \stackrel{\text { def }}{=} \sum_{i=1}^{i=f} P_{i}$. Similarly, the total number of output ports in the network (i.e. outputs of 
the third stage) is $\mathcal{O} \stackrel{\text { def }}{=} \sum_{k=1}^{k=g} Q_{k}$. The total number of links, $\mathcal{C}$, going into the middle stage is given by

$\mathcal{C} \stackrel{\text { def }}{=} \sum_{i=1}^{f} \sum_{j=1}^{m} R_{i, j}$.

Similarly, the total number of links, $\mathcal{D}$, coming out of the middle stage is given by

$\mathcal{D} \stackrel{\text { def }}{=} \sum_{j=1}^{m} \sum_{k=1}^{g} T_{j, k}$.

Therefore, the maximum number of simultaneous connections the network can support is given by

Capacity $=\min \{\mathcal{I}, \mathcal{C}, \mathcal{D}, \mathcal{O}\}$.

In other words, if $\min \{\mathcal{I}, \mathcal{C}, \mathcal{D}, \mathcal{O}\}$ exist in the network at a given time, the utilization of the network is $100 \%$ at that time.

\section{Notation.}

The set of switches in stage $i$ will be denoted by $S_{i}$. We use $C(i, j, k)$ to represent the number of connections set up between the first stage switch $i$ and the third stage switch $k$ through the middle stage switch $j$. For example, in Figure $3(\mathrm{~b}), C(1,1,1)=3$ and $C(2,1,1)=0$. We use $C(i, j, \star)$ to denote $\sum_{k=1}^{k=g} C(i, j, k)$. In other words, $C(i, j, \star)$ denotes the number of connections routed through the middle-stage switch $j$ from the first-stage switch $i$ to all switches in the third stage. Similarly, $C(\star, j, k)$ is used to denote $\sum_{i=1}^{i=f} C(i, j, k) . C(\star, j, k)$ represents the number of connections routed through the middlestage switch $j$ from all switches in the first stage to the third-stage switch $j$. For example, in Figure $3(\mathrm{~b}), C(\star, 1,1)=3$ and $C(2,2, \star)=2$.

A connection from the first stage switch $i$ to the third stage switch $k$ through the middle stage switch $j$ is often denoted by $[i, j, k]$. A connection from $i$ to $k$ should always be taken to mean a connection from an input-port of switch $i$ in stage 1 to an output port of switch $k$ in stage 3. A request for either connection or disconnection from $i$ to $k$ is indicated by $[i \rightarrow k]$. Since we only consider nearly-nonblocking operation in this paper, the terms nonblocking and nearly-nonblocking will be used synonymously in the rest of this paper.

\section{Example for rearranging connections.}

The blocking problem in a Clos network occurs due to resource conflicts. Figure 3 shows a blocking configuration in a symmetric $2 \times 2 \times 2$ Clos network. In Figure 3 (a), existing connections are shown by thick (either solid or dashed) lines. Suppose that a new connection request from input port $x$ to output port $y$ arrives. This connection from $x$ to $y$ cannot be realized without rearrangements, since all paths from the first-stage switch 1 to the third-stage switch 1 in Figure 3 are being used for other connections. However, if we rearrange the connection from input port $u$ to output $w$ (shown by dashed lines), the connection from $x$ to $y$ can be established. In Figure 3 (a), the connection from $u$ to $w$ uses switch 1 in the middle-stage. In Figure 3 (b), this connection is rearranged so that 


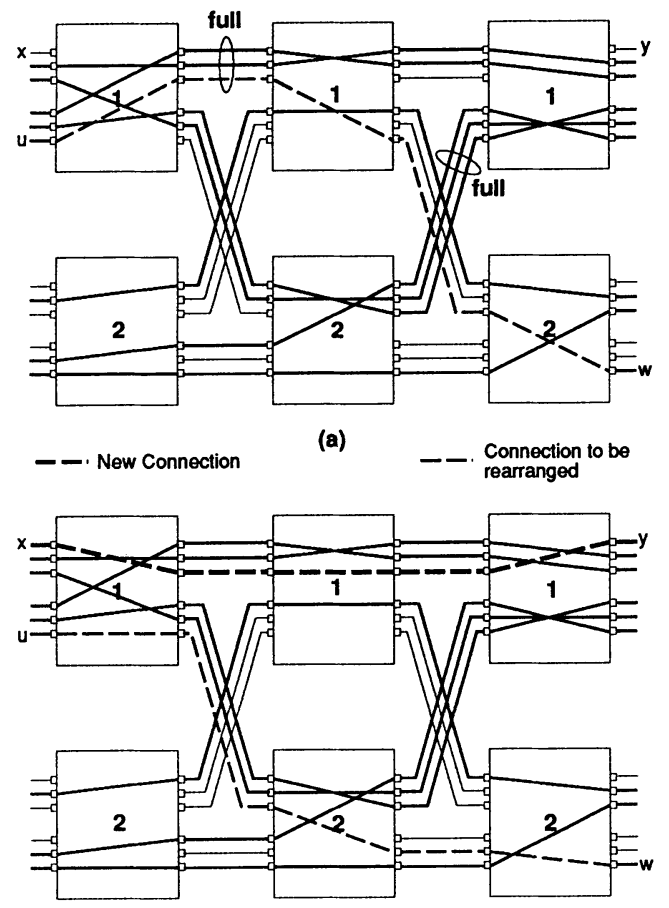

(b)

Figure 3. (a) A blocking configuration, and (b) Removal of blocking by one rearrangement.

it uses switch 2 in the middle-stage. Because of this rearrangement, the connection from $x$ to $y$ can be established (shown using thick dashed lines in Figure 3 (b)) using switch 1 in the middle-stage.

A single rearrangement does not always guarrantee that a new connection can be realized on the network. Several rearrangements may sometimes be necessary to service a new request. Moreover, each reconnection usually generates noise, and therefore affects the quality of the services provided by the network. Hence, we consider only nearlynonblocking mode of operation in this paper.

In the next two sections, we present two different control algorithms for operating the asymmetrical Clos network in the nonblocking mode. Any control algorithm that achieves nonblocking operation should specify how a new connection is realized through the network. In a three-stage network, to realize a connection from (an input port of) switch $i$ in stage 1 to (an output port of) switch $k$ in stage 3 , a switch $j$ in stage 2 needs to be found such that there are unused links from $i$ to $j$ and from $j$ to $k$. There may be several middle-stage switches $j$ which can be used to route the connection from $i$ to 
$k$; the two control algorithms presented in this paper use different policies for choosing a middle stage switch. To achieve nonblocking operation, a control algorithm, for each disconnection, must specify which connection is to be rearranged and how. The two control algorithms presented in this paper also differ in this aspect.

\section{AN ALGORITHM FOR NONBLOCKING OPERATION}

In this section, we present a control algorithm for nonblocking operation of asymmetrical Clos networks. This algorithm was originally developed by Ohta for symmetric Clos networks [17]; our contribution in this section is to derive conditions under which an asymmetrical Clos network can be made nonblocking using Ohta's control algorithm.

\section{Rules for establishing a new connection.}

To establish a new connection between the first-stage switch $I,(1 \leq I \leq f)$ and the third-stage switch $K,(1 \leq K \leq g)$, we need to find only a middle stage switch $j$ such that there is an unused channel from $I$ to $j$ and another unused channel from $j$ to $I$. Such a middle stage switch is selected using the following rule.

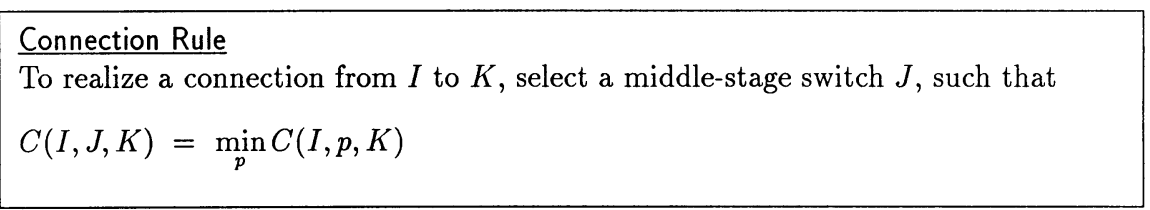

The above connection rule distributes all the connections between the first-stage switch $I$ and the third-stage switch $K$ approximately equally among all middle-stage switches, for any given $I$ and $K$. Such an even distribution of connections among all middle stage switches increases the network utilization.

\section{Rules for disconnecting an existing connection.}

Suppose that a connection $[I, J, K]$ (that is, a connection from $I$ to $K$ through the middle-stage switch $J$ ) is to be disconnected. This is accomplished using the following disconnection rule.

\section{Disconnection Rule}

1 To disconnect $[I, J, K]$, first find a middle stage switch $J_{m}$ such that $C\left(I, J_{m}, K\right)=$ $\max _{p} C(I, p, K)$.

2 if $C(I, J, K)<C\left(I, J_{m}, K\right)$ reroute a connection from $I$ to $K$ through $J_{m}$ to use the middle-stage switch $J$. In other words, a connection of the form $\left[I, J_{m}, K\right]$ is rearranged so that it becomes a connection of the form $[I, J, K]$.

3 Disconnect $[I, J, K]$.

Observe that disconnection of $[I, J, K]$ will rearrange an existing connection only if $C(I, J, K)$ is smaller than $C\left(I, J_{m}, K\right)$ by at least one; such a rearrangement ensures that the connections between the first-stage switch $I$ and the third-stage switch $K$ are divided 
approximately equally among all middle-stage switches.

Example 1 Figure 4 shows an example of a $2 \times 2 \times 2$ network with 3 links between each pair of switches in successive stages. With the network initially empty, we initiate a sequence of connection requests: $[a \rightarrow d],[c \rightarrow f]$, and $[b \rightarrow e]$. The $C(i, j, k)$ array indicates the number of connections from switch $i$ (in stage 1) to switch $k$ (stage 3 ) through the middle-stage switch $j$ at any given time. The connection from $a$ to $d$ is established (arbitrarily) through middle-stage switch 1 , since $C(1,1,1)=C(1,2,1)=0$ initially; this makes $C(1,1,1)$ one. The second connection from $c$ to $f$ is routed through the middle-stage switch 2 , since $C(1,1,1)=1$ and $C(1,2,1)=0$ after the first connection; this makes $C(1,2,1)$ one. The third connection from $b$ to $e$ can be routed through either of the middle-stage switches since $C(1,1,1)=C(1,2,1)=1$; in this figure, $b$ to $e$ is established using the middle-stage switch 1 .

A subsequent disconnection of the request from $c$ to $f$ creates imbalance for the connections from the first-stage switch 1 to the third-stage switch 1 , since $C(1,1,1)=2$ and $C(1,2,1)=0$ (see lower half of Figure 4). To restore the balance, we rearrange the connection from $b$ to $e$ such that it is routed through the middle-stage switch 2 .

The control algorithm described by the Connection and Disconnection rules presented above will be referred to as ALGO1 in the rest of this paper. We next derive the conditions under which ALGO1 achieves nonblocking operation of the asymmetrical Clos networks.

Lemma 1 In the asymmetrical Clos switching network of Figure 2, a new connection from $I$ to $K$ can always be established using the control algorithm ALGO1 if

$$
\begin{aligned}
& R_{I, j} \geq\left\lfloor\frac{P_{I}-g}{m}\right\rfloor+g \quad 1 \leq j \leq m, \quad \text { and } \\
& T_{j, K} \geq\left\lfloor\frac{Q_{K}-f}{m}\right\rfloor+f \quad 1 \leq j \leq m .
\end{aligned}
$$

Proof: Given in the appendix.

From Lemma 1, we can directly arrive at the following theorem which states the conditions for nonblocking operation of the asymmetrical Clos network using the control algorithm ALGO1.

Theorem 1 The asymmetrical Clos switching network of Figure 2 is nonblocking under the control algorithm ALGO1 if

$$
\begin{aligned}
& \min _{j}\left\{R_{i, j}\right\} \geq\left\lfloor\frac{P_{i}-g}{m}\right\rfloor+g \quad 1 \leq i \leq f, \quad \text { and } \\
& \min _{j}\left\{T_{j, k}\right\} \geq\left\lfloor\frac{Q_{k}-f}{m}\right\rfloor+f \quad 1 \leq k \leq g .
\end{aligned}
$$

Example 2 For the $2 \times 2 \times 2$ network shown in Figure 4, we notice that $P_{1}=P_{2}=Q_{1}=$ $Q_{2}=6, f=m=g=2$. In addition, $R_{i, j}=T_{j, k}=3$ for $1 \leq i, j, k \leq 2$. Hence, we have $\left\lfloor\frac{P_{i}-g}{m}\right\rfloor+g$ equal to 4 for $1 \leq i \leq 2$; since this value exceeds $\min _{j}\left\{R_{i, j}\right\}=2$, the network shown in Figure 4 is not nonblocking under the control algorithm ALGO1. However, if we change the values of $P_{1}, P_{2}, Q_{1}$ and $Q_{2}$ to 4 , this network satisfies the conditions of Theorem 1, and hence is nonblocking under the control algorithm ALGO1. 

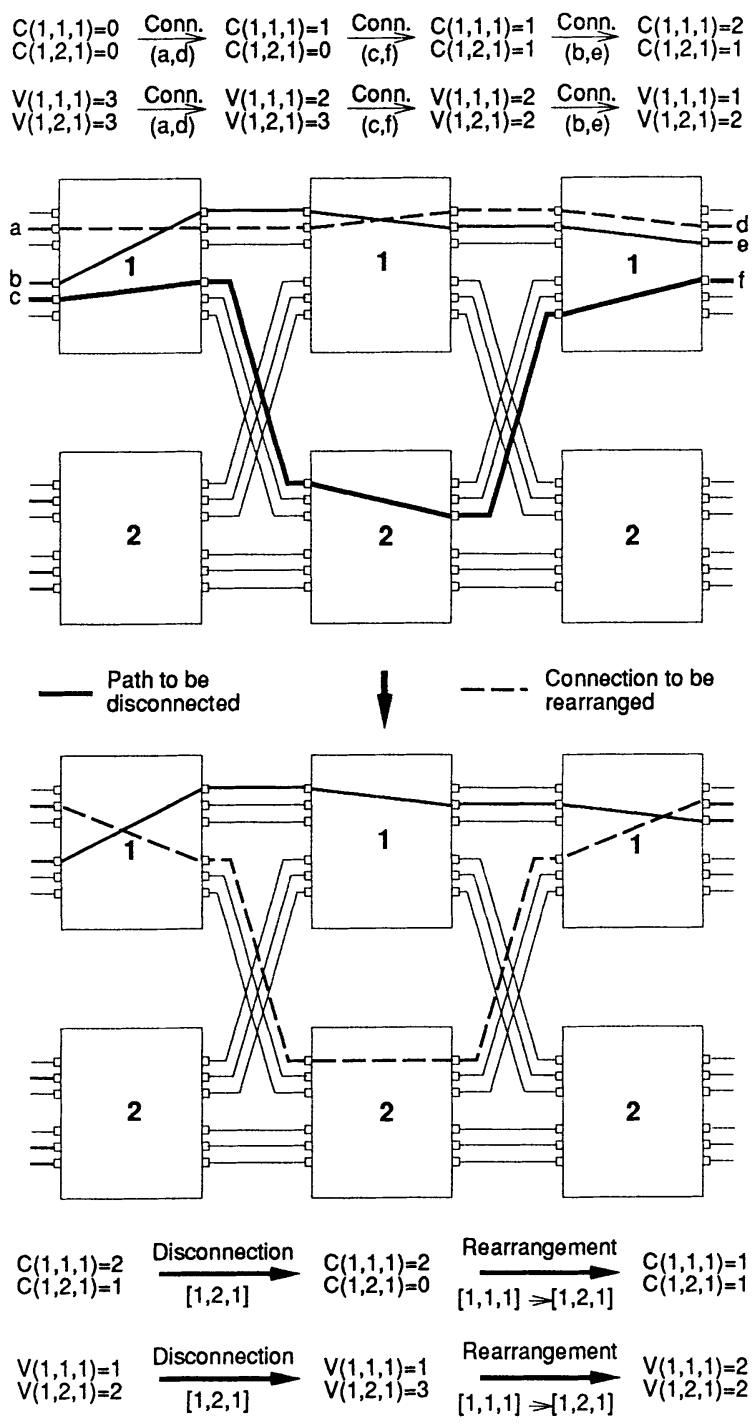

Figure 4. Example to illustrate connections and disconnections using ALGO1 and ALGO2. 


\section{A NEW ALGORITHM FOR NONBLOCKING OPERATION}

In this section, we present a different algorithm, denoted ALGO2, for achieving nonblocking operation of the asymmetrical Clos networks. Due to space limitations, we do not present analytical results on the performance of ALGO2 in this paper. However, we present a detailed comparison of ALGO1 and ALGO2 in the next section, based on simulation results. Algo2 differs from Algo1, presented in the previous section, in the way it chooses a middle stage switch for connection and disconnection. The connection and disconnection rules for ALGO2 are discussed below.

\section{Rules for establishing a new connection.}

Recall that $C(i, j, \star)$ denotes the number of connections routed through the middlestage switch $j$ from the first-stage switch $i$ to all switches in the third stage. Similarly, $C(\star, j, k)$ represents the number of connections routed through the middle-stage switch $j$ from all switches in the first stage to the third-stage switch $k$. In other words, $C(\star, j, k)=$ $\sum_{i=1}^{i=f} C(i, j, k)$ and $C(i, j, \star)=\sum_{k=1}^{k=g} C(i, j, k)$. Let the function $V(i, j, k)$ be defined as follows.

$V(i, j, k) \stackrel{\text { def }}{=} \min \left\{\left(R_{i j}-C(i, j, \star)\right),\left(T_{j k}-C(\star, j, k)\right)\right\}, \quad(i, j, k) \in S_{1} \times S_{2} \times S_{3}$.

For a given $i$ and $k, V(i, j, k)$ represents the maximum number of available paths in the network for the connection from $i$ to $k$ through middle-stage switch $j$. A middle stage switch to realize a connection from $I$ to $K$ is selected using the following rule.

\section{Connection Rule}

To realize a connection from $I$ to $K$, select a middle-stage switch $J$, such that

$$
V(I, J, K)=\max _{p} V(I, p, K)
$$

The above connection rule distributes all the connections in such a way that the available paths between a pair of first and third stage switches are uniformly distributed, to a large extent, over all the middle-stage switches. This is in contrast to ALGO1 which balances the existing connections over all the middle-stage switches. In effect, ALGO2 performs global balancing since it considers all connections of the form $[i, j, \star]$ and $[\star, j, k]$, while ALGo 1 performs only local balancing by considering only connections of the form $[i, j, k]$. Due to its global balancing property, ALGO2 can perform better for asymmetrical networks.

\section{Rules for disconnecting an existing connection.}

Suppose that a connection $[I, J, K]$ (that is, a connection from $I$ to $K$ through the middle-stage switch $J$ ) is to be disconnected. This is accomplished using the following disconnection rule. 


\section{Disconnection Rule}

1 To disconnect $[I, J, K]$, first find a middle stage switch $J_{m}$ such that $V\left(I, J_{m}, K\right)=$ $\min _{p} V(I, p, K)$.

2 if $V(I, J, K)>V\left(I, J_{m}, K\right)$ reroute a connection from $I$ to $K$ through $J_{m}$ to use the middle-stage switch $J$. In other words, a connection of the form $\left[I, J_{m}, K\right]$ is rearranged so that it becomes a connection of the form $[I, J, K]$.

3 Disconnect $[I, J, K]$.

Observe that disconnection of $[I, J, K]$ will rearrange an existing connection only if $V(I, J, K)$ is greater than $V\left(I, J_{m}, K\right)$ by at least one; such a rearrangement ensures that the available paths between the first-stage switch $I$ and the third-stage switch $K$ are divided approximately equally among all middle-stage switches.

Example 3 In Example 1, we have already explained how ALGO1 operates in the example of Figure 4. In the same figure, ALGO2 operates similarly, but instead uses the values of $V(I, J, K)$ for choosing the middle-stages switches for connections and disconnections. For the sequence of connections $[a \rightarrow d],[c \rightarrow f]$, and $[b \rightarrow e]$, the middle-stage switches chosen by Algo 2 are the same as those chosen by Algo1, although Algo1 and Algo2 use different criteria. Connections of the type $[2,1,1]$ or $[1,1,2]$ interfere with $[1,1,1]$ connections by reducing $V(1,1,1)$, although they do not affect $C(1,1,1)$. A more complex example in which AlgO1 and ALGO2 behave differently is given in Example 4.

Example 4 We next present an example to show a situation in which ALGO1 leads to blocking while ALGO2 does not.

Figure 5 illustrates a case where ALGO2 makes a better routing choice than ALGO1. Assume a $2 \times 2 \times 2$ Clos network with faults. The number of available links (after removing the faulty links) is given by the $R_{i, j}$ and $T_{j, k}$ matrices.

$\left[R_{i, j}\right] \stackrel{\text { def }}{=}\left[\begin{array}{ll}3 & 1 \\ 3 & 3\end{array}\right] \quad$ and $\quad\left[T_{j, k}\right] \stackrel{\text { def }}{=}\left[\begin{array}{ll}3 & 0 \\ 3 & 3\end{array}\right]$

A sequence of three connection requests

$[1 \rightarrow 1], \quad[1 \rightarrow 1], \quad[1 \rightarrow 2]$

is applied to both algorithms and their states are shown in the two tables of Figure 5.

The state of ALGO1 is given by $C(I, J, K)$, which denotes the number of established connections of the type $[I, J, K]$. The numbers in italics indicate the available connections through switches $I, J, K$. The state of ALGO2 is given by $V(I, J, K)$. The choices made by each algorithm are indicated by the check-marks in Figure 5 . The data used by the algorithms are indicated by ovals, and correspond to the two middle-stage switches. A tracing of the algorithms for each request is given below.

$[1 \rightarrow 1]$ : (see state S1) Algo1 arbitrarily picks the first middle-stage switch. Algo2 selects the same switch, but because of higher availability of connections (3 vs 1). The newly established connection changes the state of each algorithm from S1 to S2. 


\begin{tabular}{|c|c|c|c|c|c|c|c|}
\hline \multicolumn{3}{|c|}{ Algo1 } & \multicolumn{5}{|c|}{ (Established connections) } \\
\hline & $\mathbf{J}$ & $\mathbf{K}$ & S1 & $\mathbf{S}$ & & S3 & 3 \\
\hline 1 & $\begin{array}{l}1 \\
2\end{array}$ & 1 & $\left(\begin{array}{l}0 \\
0\end{array}\right.$ & $\left(\begin{array}{l}1 \\
0\end{array}\right.$ & & & $\begin{array}{l}2 \\
0\end{array}$ \\
\hline 1 & $\begin{array}{l}1 \\
2\end{array}$ & 2 & $\begin{array}{l}\mathbf{0} \\
\mathbf{0}\end{array}$ & $\begin{array}{l}0 \\
0\end{array}$ & & & $\int_{0}^{0}$ \\
\hline 2 & $\begin{array}{l}1 \\
2\end{array}$ & 1 & $\begin{array}{l}0 \\
0\end{array}$ & & $\begin{array}{l}2 \\
3\end{array}$ & & $\begin{array}{l}2 \\
2\end{array}$ \\
\hline 2 & $\begin{array}{l}1 \\
2\end{array}$ & 2 & $\begin{array}{l}0 \\
0\end{array}$ & $\begin{array}{l}0 \\
0\end{array}$ & $\begin{array}{l}0 \\
3\end{array}$ & & $\begin{array}{l}0 \\
3\end{array}$ \\
\hline
\end{tabular}

Algo2 (Remaining available connections)

\begin{tabular}{|c|c|c|c|c|c|}
\hline I & $\mathbf{J}$ & S1 & S2 & S3 & S4 \\
\hline 1 & $\begin{array}{l}1 \\
2\end{array}$ & $\left(\begin{array}{l}3 \\
1\end{array}\right)$ & & $\begin{array}{l}1 \\
1\end{array}$ & $\begin{array}{l}1 \\
0\end{array}$ \\
\hline 1 & $\begin{array}{l}1 \\
2\end{array}$ & $\begin{array}{l}0 \\
1\end{array}$ & $\begin{array}{l}0 \\
1\end{array}$ & 0 & $\begin{array}{l}0 \\
0\end{array}$ \\
\hline 2 & $\begin{array}{l}1 \\
2\end{array}$ & $\begin{array}{l}3 \\
3\end{array}$ & $\begin{array}{l}2 \\
3\end{array}$ & $\begin{array}{l}1 \\
3\end{array}$ & $\begin{array}{l}1 \\
3\end{array}$ \\
\hline 2 & $\begin{array}{l}1 \\
2\end{array}$ & $\begin{array}{l}0 \\
3\end{array}$ & $\begin{array}{l}\mathbf{0} \\
\mathbf{3}\end{array}$ & $\begin{array}{l}0 \\
3\end{array}$ & $\begin{array}{l}0 \\
2\end{array}$ \\
\hline
\end{tabular}

$$
\begin{aligned}
& \text { S1 } \\
& 1 \stackrel{1}{\rightarrow}-1 \\
& \text { S2 } \\
& 1 \stackrel{2}{\rightarrow}-1 \\
& \text { S3 } \\
& 1 \stackrel{?}{\rightarrow}-2 \\
& \text { (blocked) }
\end{aligned}
$$

$$
\begin{gathered}
\stackrel{\substack{S 1 \\
1}}{\rightarrow} 1 \\
S 2 \\
1 \stackrel{1}{\rightarrow} 1 \\
S 3 \\
1 \stackrel{2}{\rightarrow} 2 \\
S 4
\end{gathered}
$$

Figure 5. Traces of the two algorithms; a case for ALGO2.

$[1 \rightarrow 1]$ : (see state S2) This time, Algo1 chooses to route through the second middlestage switch, in an attempt to uniformly distribute the connections from $I=1$ to $K=1$ over all middle-stage switches. Instead, ALGO2 selects the first switch because it still has higher availability of connections (2 vs 1). The new state is S3.

$[1 \rightarrow 2]$ : (see state S3) For Algo1, there are no available paths to establish this connection. Algo2, however, can route it through the second middle-stage switch.

The above example shows the difference between the two algorithms. Algol selects with local balancing criteria and ignores the number of available through the network. ALGO2 decides based on global balancing criteria and uses this availability information. In asymmetrical networks, this difference is critical and gives ALGO2 the advantage.

\section{SIMULATION RESULTS}

In this section, we present a comparative evaluation of the two algorithms ALGO1 and AlgO2 when applied on asymmetrical Clos networks. The previous two sections have presented conditions under which ALGO1 and ALGO2 achieve nonblocking operation of 
an asymmetrical network. In this section, we study a different measure: the extent to which a given network can be utilized without introducing blocking using algorithms AlgO1 and AlgO2. We next describe the simulator and the performance measures used to evaluate the algorithms.

\subsection{Simulator and performance measures Simulator.}

A simulator was developed to simulate three-stage asymmetrical Clos networks with or without faults. Different routing algorithms can be evaluated by keeping the network configuration, fault-patterns and the sequence of connections and disconnections fixed. A sequence of connections is generated randomly with a uniform distribution over the available input (first-stage) and output (third-stage) switches. Connections and disconnections are generated so that a predefined average load is maintained. When the average number of requests generated during any given time interval equals the capacity of the network (see (1)), it corresponds to $100 \%$ load.

Faults are generated by randomly selecting links in the network and deleting them. Switching element faults can be simulated by making all links incident on the switching element faulty. If a link connecting an input port (respectively, output port) to a switch in stage 1 (respectively, stage 3 ) is faulty, that port ceases to exist and the capacity of the network is correspondingly altered. The desired number of faults is specified as a percentage of the network's capacity given in (1). In the experiments presented in this section, we varied the fault percentage from $0 \%$ to $20 \%$ of its full capacity.

\section{Performance measures.}

A good control algorithm in general distributes the connections among the links of the network such that the switch utilization is maximized and the blocking probability is minimized. The measures we use to compare control algorithms are described below.

Measure 1: (M1) We count the number of established connections until the first blocking. No disconnections or rearrangements are performed; hence, even if a blocked request can be serviced with one or more rearrangements, it is still considered a blocked request. The larger the result of this measure is, the better the algorithm is in establishing connections.

Measure 2: (M2) We count the number of blocked requests until a certain fraction of the network capacity is utilized. Usually we measure the number of blocked requests until the network capacity is utilized $100 \%$. However, for certain networks and control algorithms, it may not be possible to achieve $100 \%$ utilization of the network; in such cases, we set the utilization goal to a smaller value. A low value for this measure indicates that the algorithm reduces the blocking probability for connections.

Measure 3: (M3) Here, we count the number of blockings occurred while servicing a certain number of requests, which include connections and disconnections. In order to observe the effect of disconnections and rearrangements, the total number of requests must be much larger than the capacity of the network. A low value for this 


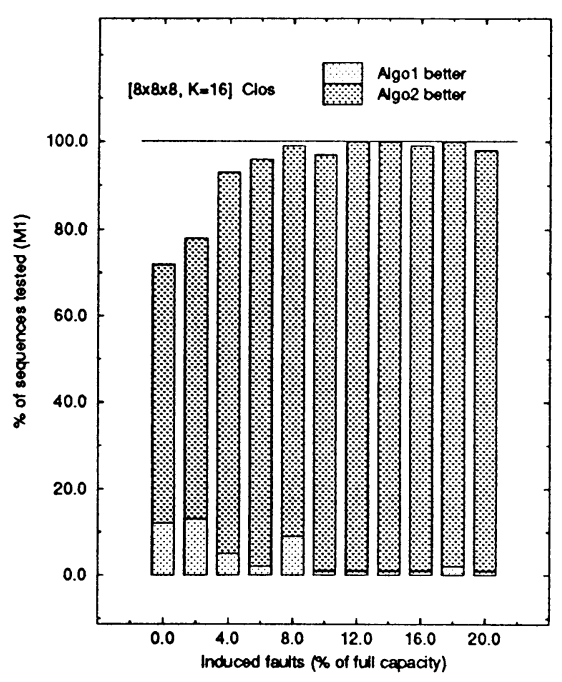

(a)

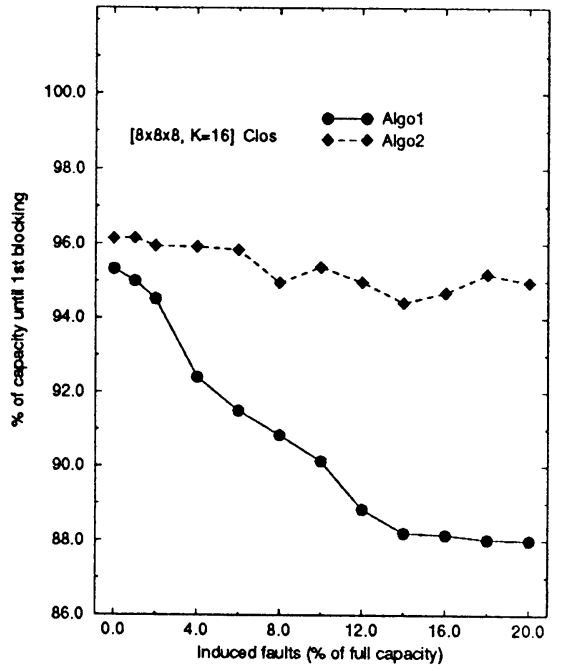

(b)

Figure 6. Comparison of the two algorithms for configuration 1 based on M1.

measure indicates that the algorithm is highly effective in realizing connections and disconnections.

The above three measures can be used to evaluate and compare the performance of routing algorithms Algo1 and AlgO2 for different load conditions and fault-patterns.

\subsection{The Results}

We will present and analyze simulation results for the following two configurations.

Configuration 1. $f=m=g=8$ and $R_{i, j}=T_{j, k}=K=16, \quad 1 \leq i, j, k \leq 8$. That is, each stage consists of 8 switches, and each pair of switches in successive stages is connected by 16 links. Further, the number of inputs to each first stage switch is 128 , and the number of outputs of each third-stage switch is also 128.

Configuration 2. $f=m=g=16$ and $R_{i, j}=T_{j, k}=K=8,1 \leq i, j, k \leq 16$. That is, each stage consists of 16 switches, and each pair of switches in successive stages is connected by 8 links. Further, the number of inputs to each first stage switch is 128 , and the number of outputs of each third-stage switch is also 128 .

The first configuration represents a network capacity of 1024 connections and the second one a capacity of 2048 connections.

\subsubsection{Results for configuration 1}




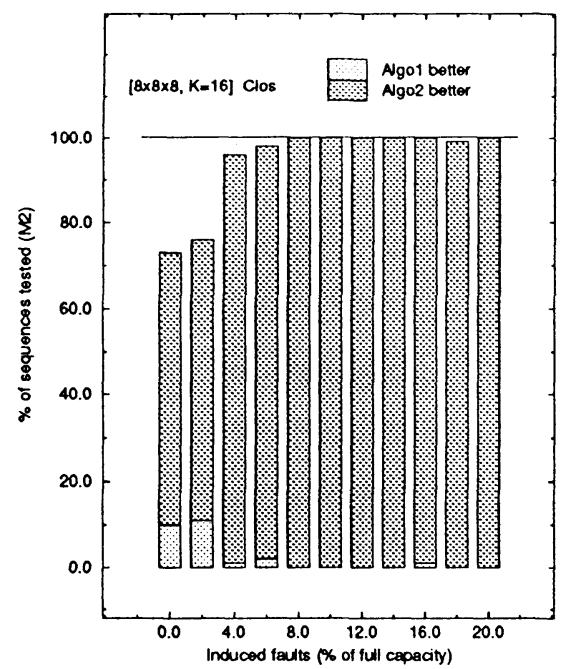

(a)

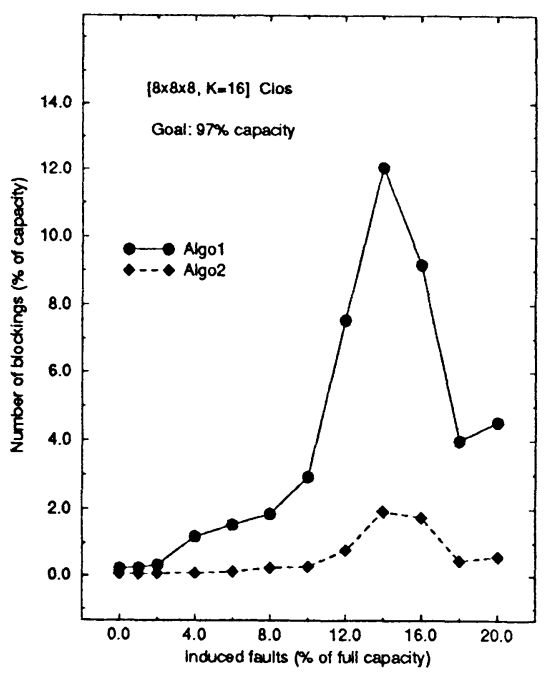

(b)

Figure 7. Comparison of the two algorithms for configuration 1 based on M2.

\section{Performance with respect to M1.}

Figure 6(a) shows a comparison between Algo1 and Algo2, based on the first measure. Five hundred different random sequences of requests for connections have been tested, and the algorithms were compared according to the maximum capacity they could utilize until the first blocking occurred. In a fault-free network, ALGO2 did (strictly) better in $60 \%$ of the cases, worse in $12 \%$, and both algorithms performed equally in the remaining $28 \%$. As more and more link-faults are introduced in the network, (thus making it more asymmetrical) ALGO2 outperformed Algo1 as predicted. With more than $10 \%$ faults, Algo 2 did better than Algo1 in more than $95 \%$ of the cases.

Figure 6(b) shows the number of connections in the network as a percentage of its capacity at the time of first blocking (M1). We observe that, as the number of faults varies from $0 \%$ to $20 \%$ of its full capacity, ALGO2 deteriorates only by $2 \%$. For the same sequences and fault-patterns, the performance of ALGO1 drops by nearly $7 \%$.

\section{Performance with respect to M2.}

Figure 7(a) shows a comparison of the two algorithms for the second measure (M2), using the same 500 random input sequences as above. With more than $4 \%$ faults in the network, ALGO2 performed better in more than $95 \%$ of the cases. With more than $8 \%$ faults in the network, ALGO2 outperformed ALGO1 in at least $99 \%$ of the cases.

Figure 7 (b) shows the average number of blockings for the two algorithms until the 
number of connections in the network reached $97 \%$ of the capacity of the network ${ }^{2}$. From this figure, we conclude that ALGO1 causes 4 to 12 times more blocked requests than ALGO2 as the number of faults increases above $12 \%$ of the network capacity.

The "sawtooth" spikes observed in all the graphs can be explained as follows. The fault patterns were generated using the same random-number seed in all our experiments. In every experiment we initialized the random number generator with this seed. Therefore, for example, $14 \%$ faults include the same $12 \%$ faults as in the $12 \%$ fault case, plus an additional set of $2 \%$ faults. Initially, as new faults are added to the network, certain regions of the network are affected more, because of concentrated faults. However, after a certain number of faults in the network, almost each region is affected equally with the addition of new faults; this causes an improvement in network performance.

In general, concentrated faults result in performance degradation. This degradation is more intense in ALGO1, because ALGO1 does not adapt well to asymmetrical networks. By design, ALGO2 adapts better to asymmetrical networks, and therefore, its performance is always less sensitive to irregular fault patterns.

\section{Performance with respect to M3.}

In the third measure, (see Figure 8), we tested both algorithms with random input sequences of 10,000 requests, which included both connections and disconnections. The utilization was kept at $90 \%$ of the capacity. For configuration 1 , as the number of faults was varied from $0 \%$ to $20 \%$ of full capacity, the blocking probability for ALGO2 almost always remained under 0.001 . On the other hand, the blocking probability for Algo1 rose almost linearly, upto 0.075 , for the same experiments. Configuration 2 , having double the capacity, experienced higher blocking probability (approximately double, with fluctuations, as the number of faults increased beyond $12 \%$ ).

The results in these two graphs are averages over ten different random input sequences, which were applied in common for both algorithms. The $t$-test, performed on these results, indicated that in all cases with faults, ALGO2 performed better than ALGO1 with $99.9 \%$ certainty. In the case of $0 \%$ faults the certainty was between $95 \%$ and $99 \%$.

\subsubsection{Results for configuration 2}

Figures $9,10,8(\mathrm{~b})$ are for configuration 2 , and correspond to figures $6,7,8(\mathrm{a})$, respectively, of the previous configuration. Recall that the second configuration has twice the capacity of the first configuration. The results for the second configuration with respect to measures 1 and 2 are similar to those for the first configuration. From figures 8 (a) and (b), we conclude that each algorithm experiences more blocked requests for configuration 2 than for configuration 1. From figures 6 (b) and 9 (b), we conclude that the percentage of network capacity reached before the first blocking is lower for the second configuration than for the first configuration. However, for each measure, ALGO2 outperforms AlGO1 for configuration 2 .

\footnotetext{
${ }^{2}$ The capacity of the network is determined after removing the faulty components.
} 


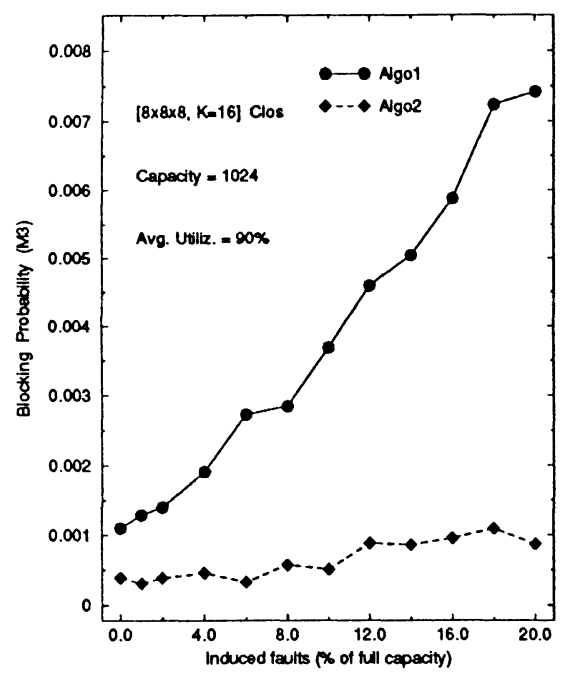

(a)

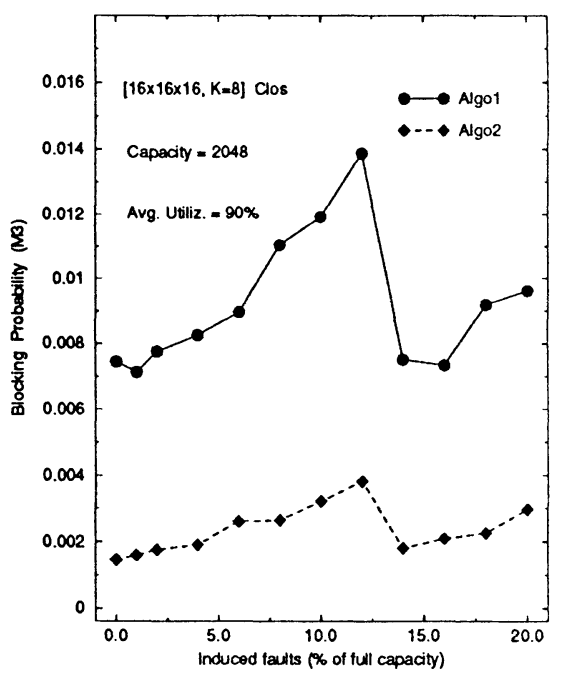

(b)

Figure 8. Comparison of the two algorithms for the 2 configurations based on M3.

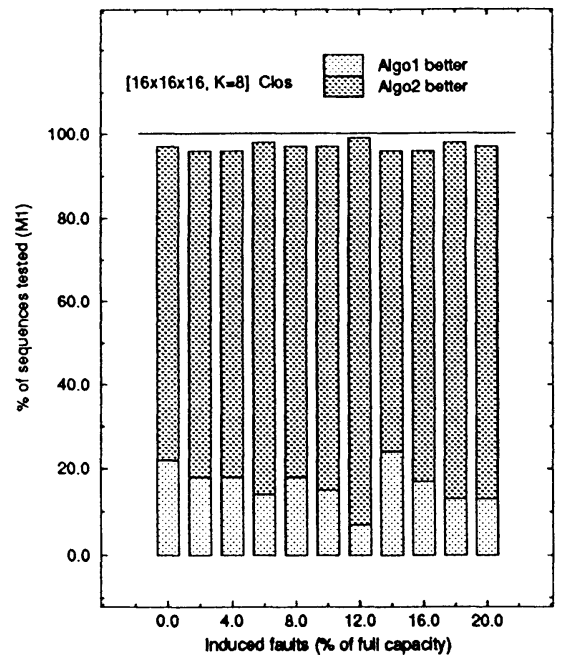

(a)

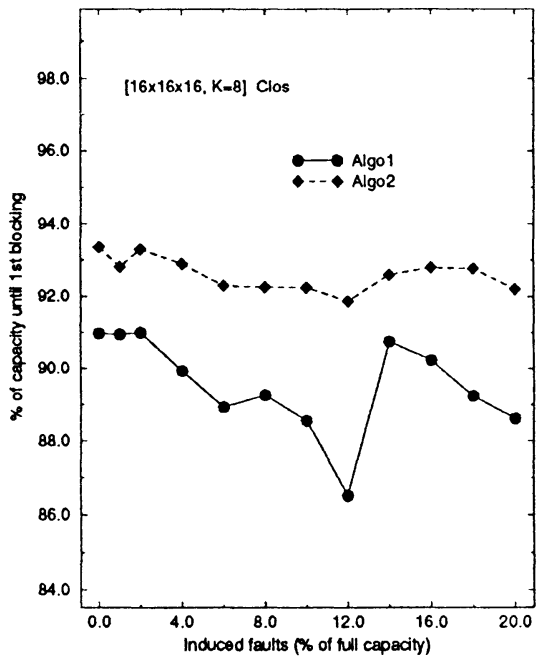

(b)

Figure 9. Comparison of the two algorithms for configuration 2 based on M1. 


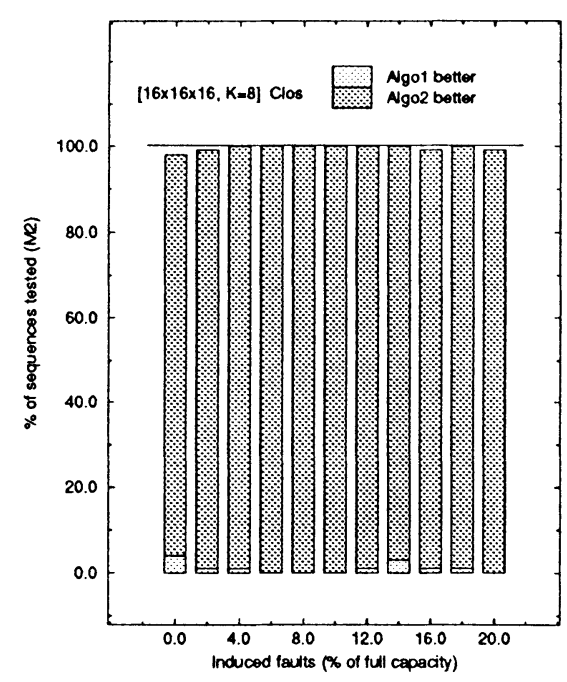

(a)

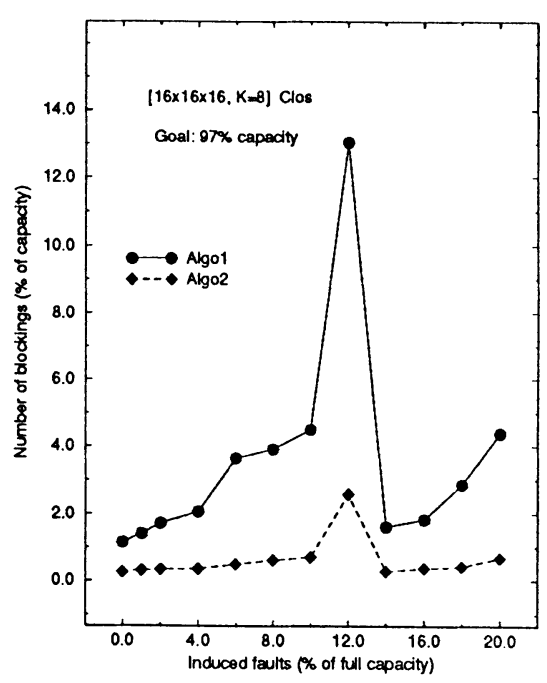

(b)

Figure 10. Comparison of the two algorithms for configuration 2 based on M2.

\section{A QUEUEING MODEL FOR THE ROUTER}

Figure 11 shows a general circuit switching mechanism. A set of input ports (or switches) are interconnected with a set of output ports (or switches) through a switching network.

Initially, let us assume that an input switch $J$ is connected to an output switch $K 2$ and data flows through the established connection. At some point in time, the incoming stream decides to terminate the connection, and it issues a disconnection request. The request is processed (in a centralized or distributed fashion) and the port is disconnected by the router. A subsequent connection request from the same switch to an output switch $K 1$ will first issue a connection request. During the time the incoming request is processed by the router, the incoming data stream may be temporarily buffered. As soon as a routing decision is reached, the path is established and the buffered data stream is transmitted to the output switch.

We model the above scheme with the mixed queuing network shown in Figure 12. It consists of an open subnetwork and a closed subnetwork, which interact indirectly - that is, without exchanging customers. In this paper we will only model and quantitatively analyze the closed subnetwork. We will also briefly discuss how the performance of this subsystem affects the design choices for the open subsystem. 


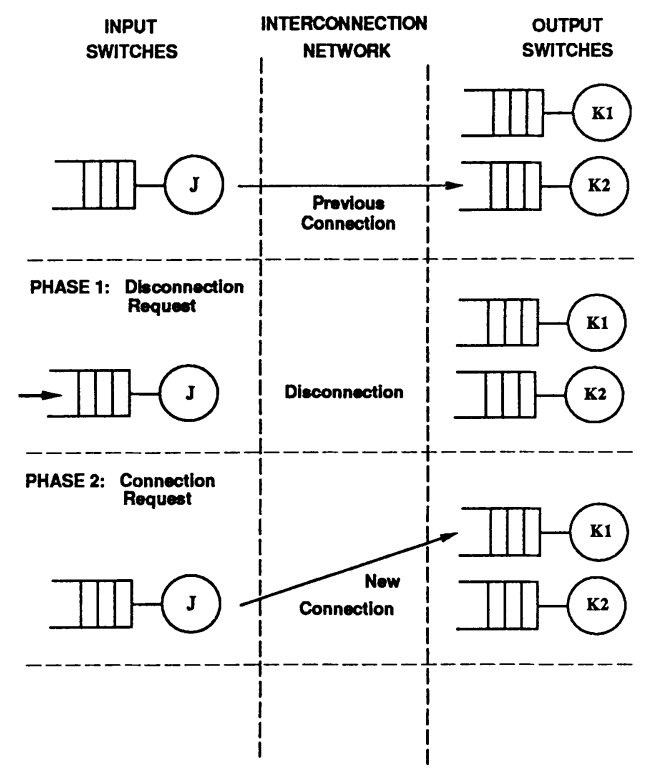

Figure 11. Connections and disconnections in a Circuit-Switched Network.

\subsection{The closed queuing network}

Each input port of our switching network is a customer in the closed subsystem. Assuming one-to-one connections, the maximum number of simultaneous connections is equal to the number of input ports. Let this number be $N$.

The closed subsystem consists of four service centers, \{ConN, CREQ, IDLE, DREC , (see Table 1), and $N$ customers. Centers $\{$ CREQ, DREQ $\}$ involve queuing, and centers \{CONN, IDLE\} involve pure delay without any queuing. Delay servers are modeled as $N$ parallel servers with the same service rate. There is a single class of customers with different service demands at each center.

Each center $i$ has an average population $Q_{i}$ (queue-size), a server utilization $U_{i}$, and a Response time $R_{i}$, (which includes the service and queuing delays at this center). All customers have a service demand $D_{i}$ at center $i$. Also, let the throughput of the closed system be $X$.

The life-cycle of a customer starts at center IDLE. Initially, an input port is disconnected. As soon as a connection request arrives, the disconnected customer moves to the queue of the CREQ server. After the request is serviced and a routing decision is reached, the customer goes to the CoNN delay server for the duration that the connection is maintained. At the end of the connection, a disconnection request is issued and the customer is queued behind the DREQ server. When the disconnection is established, the customer returns to the IDLE center and completes the cycle. 
Table 1

Service-Centers in the Closed subsystem.

\begin{tabular}{cccc}
\hline Center name & Type & Associated measures & Customer type \\
\hline IDLE & Delay & $Q_{\text {Idle }}, R_{\text {Idle }}$ & Idle Port \\
CREQ & Queuing & $Q_{C R e q}, D_{C R e q}, R_{C R e q}, U_{C R e q}$ & Port Requesting Connection \\
CONN & Delay & $Q_{C o n n}, R_{C o n n}$ & Connected Port \\
DREQ & Queuing & $Q_{D R e q}, D_{D R e q}, R_{D R e q}, U_{D R e q}$ & Port Requesting Disconnection \\
\hline$Q_{i}:$ Queue-size, $\quad D_{i}:$ Service-demand, $R_{i}:$ Residence-time, & $U_{i}:$ Utilization \\
\hline
\end{tabular}

Since we have assumed a centralized server, both connection and disconnection requests must be serviced by the same server. In order to make the model easier to solve, we chose to use a single class of customers and two shadow servers, rather than two separate servers and two classes of customers. This choice reduces the complexity of the exactMVA algorithm, as will be explained in the next section.

Qualitatively, we can think of connections and disconnections as two types of customers, which have possibly different service demands from the centralized router. We assume two different queues, one for connections and one for disconnections. The reason for this is that we do not want to penalize the customers with the smaller service demand, by forcing all of them to wait in a single queue for the same (average) amount of time. In this way, we achieve smaller average response times for the customers with smaller service demands. The two queues are assumed to be FIFOs of adequate size, and the router is assumed to alternate between them with equal priority.

\subsection{The open queuing network}

The data transfer (ATM packets, for example) is assumed to flow from the input port to the output port, with buffering at both ends. The double buffering is required to smooth the variation in the open network's throughput, (that is, to achieve approximately constant transmission rates). The input buffer is modeled as a FIFO queuing server, which has a service rate higher than that of the output buffer's server. This way, the fast server of the input buffer acts as an internal pump, which buffers and pumps the data at higher speeds inside the switch (see Figure 12). This is done in order to reduce the overhead of the router in decision making, by buffering the incoming data stream for some period of time before and after a connection or disconnection is established.

Since data transfer and routing can be overlapped with the decision-making process of the router (see Figure 13), and since this overlap is protocol dependent, we do not model a direct interference between the closed subsystem (which models the router's decisionmaking) and the open subsystem (which models the data transfer). Instead, we assign a master role to the closed subsystem and a slave role to the open subsystem. The response times at the queuing centers of the closed network may result in possible data queuing in the open network, depending on the overlap between the routing and the data-transfer processes. 


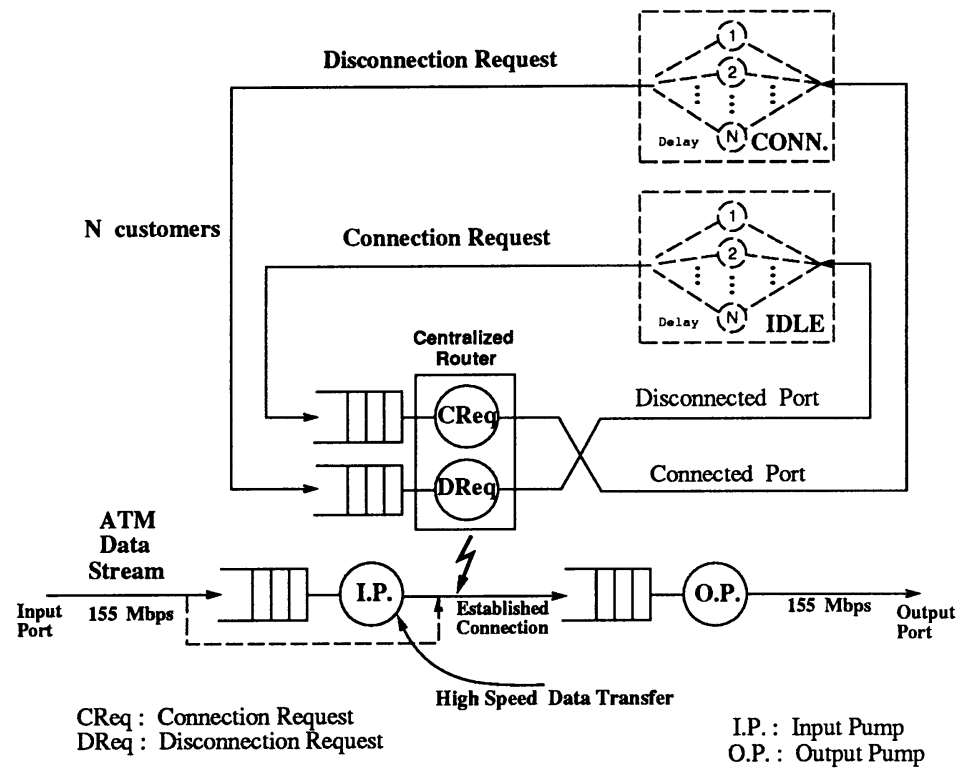

Figure 12. A queuing model for a semi-centralized router in a circuit switching network.

\subsection{An Exact-MVA Algorithm}

To quantitatively evaluate the performance of the router in the steady-state, we use Mean Value Analysis (MVA) to solve our queuing network [14,11]. Since we have assumed a single class of customers, solving the closed network with an exact-MVA method is feasible and very efficient.

An exact-MVA algorithm computes the throughput and the response time of the router. To achieve this, it uses an iterative method which computes the average queue lengths for $n$ customers, given the average queue lengths for $n-1$ customers. The exact-MVA method solves the closed-susbsystem model accurately. However, the accuracy of the solution for the real system depends primarily on the accuracy of the parameterization of the model and our modeling assumptions. The algorithm that we used for exact-MVA is presented

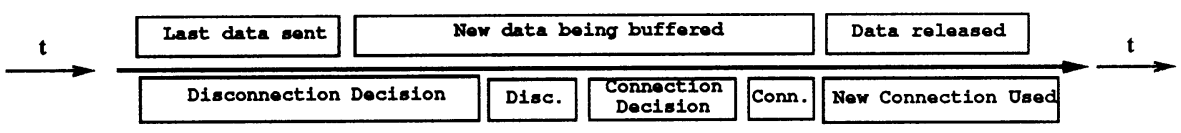

Figure 13. Overlapping of data-transmission and router's decision-making in a circuit switched network. 
in Figure 14.

\section{Exact Mean Value Analysis Algorithm for the Closed subnetwork}

1. Initialization : $\quad Q_{\text {Idle }}=Q_{C R e q}=Q_{C o n n}=Q_{D R e q}=0 ; \quad X=0 ;$

2. for $\mathrm{k}=1$ to $N$ do

2.1. Compute the queuing-center utilizations:

$U_{C R e q}=X \cdot D_{C R e q}$;

$U_{C R e q}=X \cdot D_{C R e q}$;

2.2. Compute the queuing-center residence times:

$R_{C R e q}=D_{C R e q} \cdot\left(1+Q_{C R e q}\right) /\left(1-U_{D R e q}\right) ;$

$R_{D R e q}=D_{D R e q} \cdot\left(1+Q_{D R e q}\right) /\left(1-U_{C R e q}\right) ;$

2.3. Compute the throughput from Little's Law:

$X=k /\left(R_{\text {Conn }}+R_{\text {CReq }}+R_{\text {Idle }}+R_{D \text { Req }}\right)$

2.4. Compute the average queuing at each center $i$ :

$Q_{i}=X \cdot R_{i} ; \quad i \in\{$ IDLE, CREQ, ConN, DREQ $\}$

3. end ; Repeat, for all $N$ customers.

Figure 14. An exact-MVA algorithm, which solves the proposed queuing model.

The average residence times for the delay centers are constant and need not be computed in every iteration. As mentioned earlier, the connection and disconnection service centers are interdependent with equal priority; these centers model the time-sharing operation of the router to service connection and disconnection requests. The algorithm captures this effect by inflating the service demands of each type of customers (e.g., connections) with the remaining amount of available service (which is obtained after subtracting the amount of service required by the other type of customers (e.g., disconnections)).

The algorithm described above operates by injecting one customer to the system in every iteration, and computing its "steady-state distribution" over the closed network's centers. As soon as the entire population is injected, the model is considered solved. Thus, this algorithm runs in $\mathcal{O}(N)$ time and is, therefore, very fast and efficient.

\section{PERFORMANCE RESULTS}

In order to validate our model and evaluate the accuracy of the solution method that we used, we developed an event-driven simulator and gathered simulation results for several test cases. 
We simulated a centralized router, which services two FIFO queues with equal priority. In our preliminary results, we considered constant service demands for connections and disconnections. All input ports are initially set to be idle for an exponentially distributed amount of time. Then, they request connections from the router, and their requests are serviced in an FCFS fashion. The router (server) alternates between its two queues. If one queue is empty, then the other is selected until the former receives a customer. After the router services a customer's request, the connection remains established for an exponentially distributed amount of time, until it issues a disconnection request.

Our simulator measures the router-throughput, which is the average number of connections and disconnections serviced by the router per unit time, the customer distribution (i.e. queue sizes and utilizations) at the various delay and queuing centers of the system, response times for connection and disconnection requests, and more.

The inputs to the simulator (and to our model) are described below. The numbers in parentheses are the parameters we used for the results presented in this paper, and are taken from a proposed router implementation in VLSI, which is currently under development by our research group.

- The number of input ports, $(N=64, \ldots, 1024)$, which represents the total capacity of the switch, assuming one-to-one connections only.

- The constant connection and disconnection request service times, $\left(D_{C R e q}=300\right.$ nsec and $D_{D R e q}=500 \mathrm{nsec}$, respectively).

- The average duration of an established connection between two ports, $\left(D_{C o n n}=\right.$ $R_{\text {Conn }}=500,000 \mathrm{nsec}$ ).

- The average amount of time an input port remains idle, $\left(D_{\text {Idle }}=R_{\text {Idle }}=55,556\right.$ nsec, for a target capacity utilization of $90 \%)$.

In the following, we present the simulation results for the above set of input parameters, and we compare them against our model's predictions. In all graphs presented here, we varied the capacity of the network (i.e., the number of input ports) from 64 to 1024 . The simulation experiments were run for $100 \mathrm{msec}$ of simulation time.

In Figure 15(a), we observe that the throughput of the router is linear up to 640 ports and saturates to 1.25 (connections and disconnections per $\mu \mathrm{sec}$ ) after that point. The error of our model, with respect to the simulation results, is constantly around $0.5 \%$.

Figure 15(b) shows the capacity utilization (percent of full capacity) for each network configuration. As the router's throughput saturates and the demand for service by the router increases, more customers are waiting for service, rather than being idle or connected. Therefore, the utilization of the network's capacity decreases. The two graphs in Figure 16 show the distribution of the status - which can be idle or connected - of the input ports. The error of our model for these graphs does not exceed $2 \%$.

Figures 17 and 18 show the router's response time for connection and disconnection requests, respectively. Graphs $17(\mathrm{a})$ and 18(a) are magnifications of graphs $17(\mathrm{~b})$ and 18(b), respectively. We observe that the error in these graphs is significant, especially for the disconnections. This error becomes larger at higher network capacities, as the router begins to saturate. 
Throughput vs. Capacity

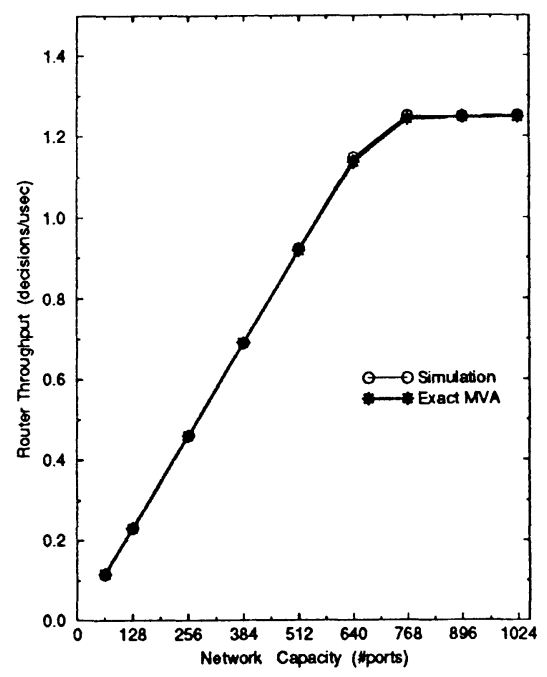

(a)
Capacity Utilization

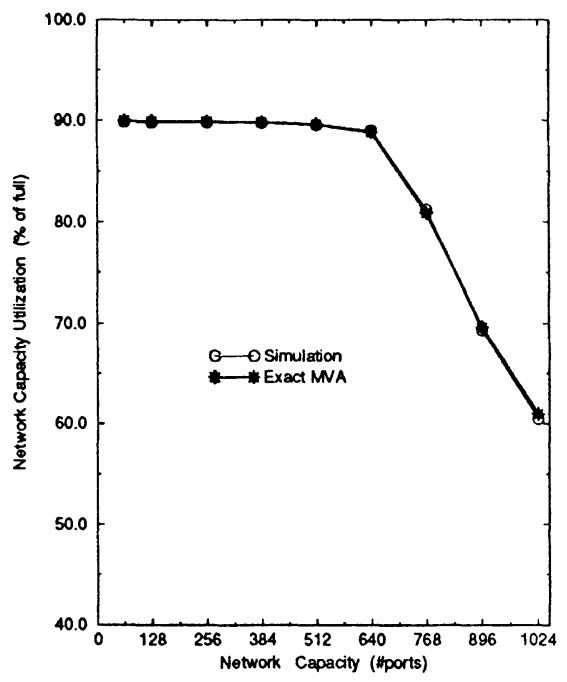

(b)

Figure 15. Throughput and Capacity Utilization of the Network

Capacity Utilization

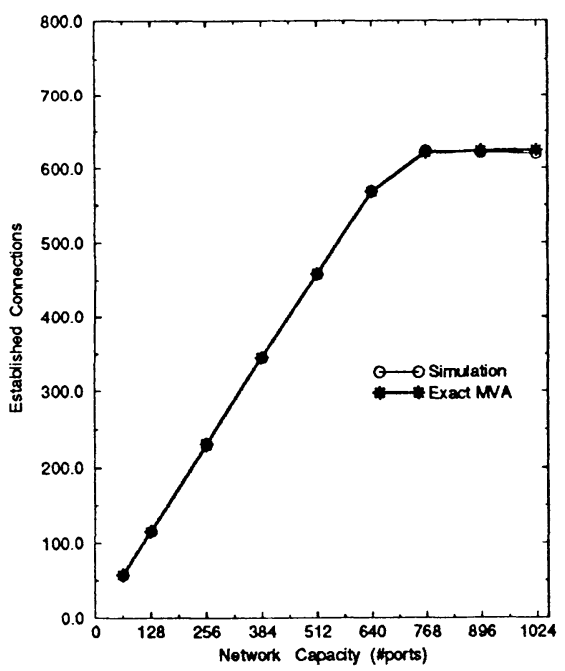

(a)
Unused Capacity

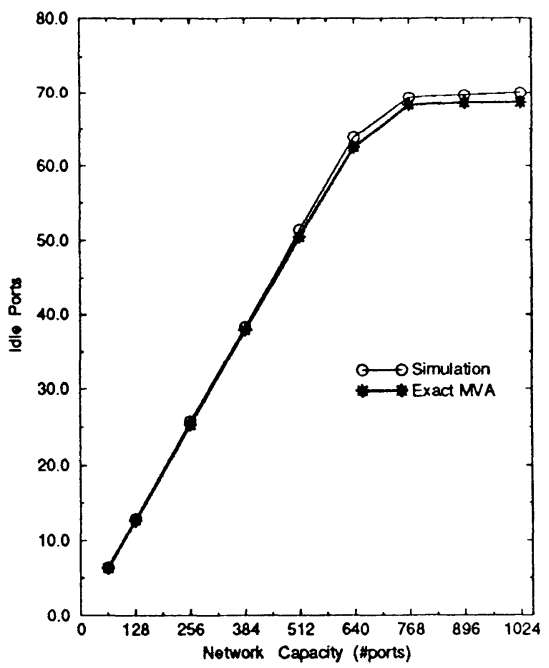

(b)

Figure 16. Number of Connected and Idle ports 
Router Response Time

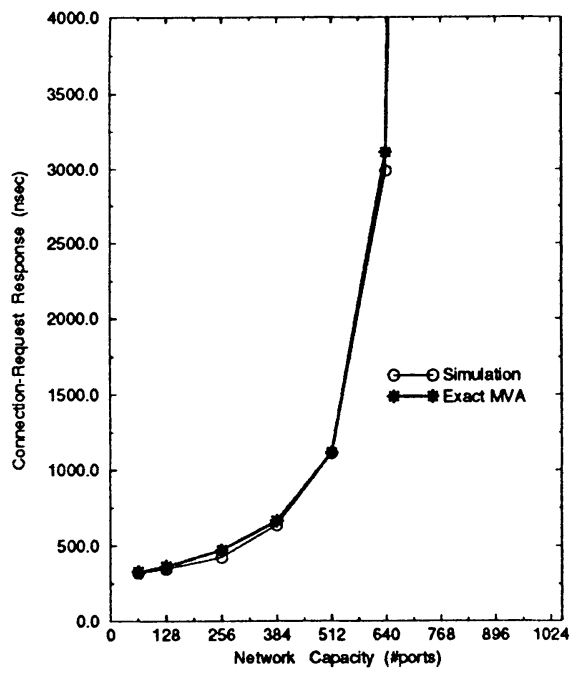

(a)
Router Response Time

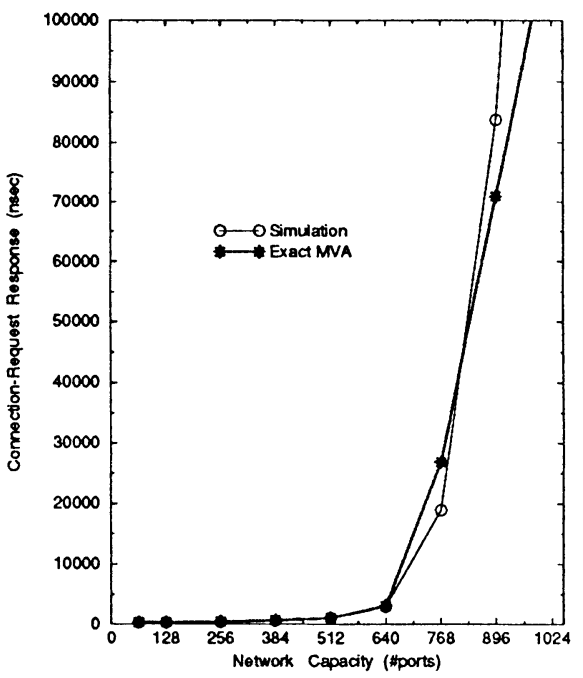

(b)

Figure 17. Router Latency for Connection-Requests

Router Response Time

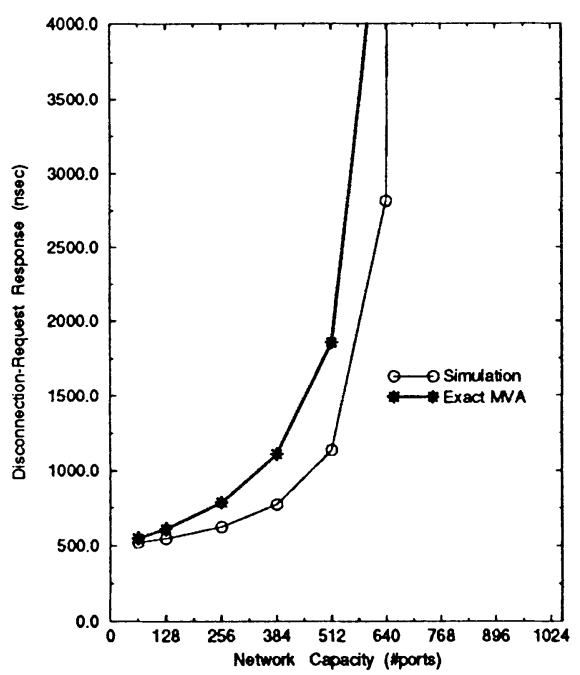

(a)
Router Response Time

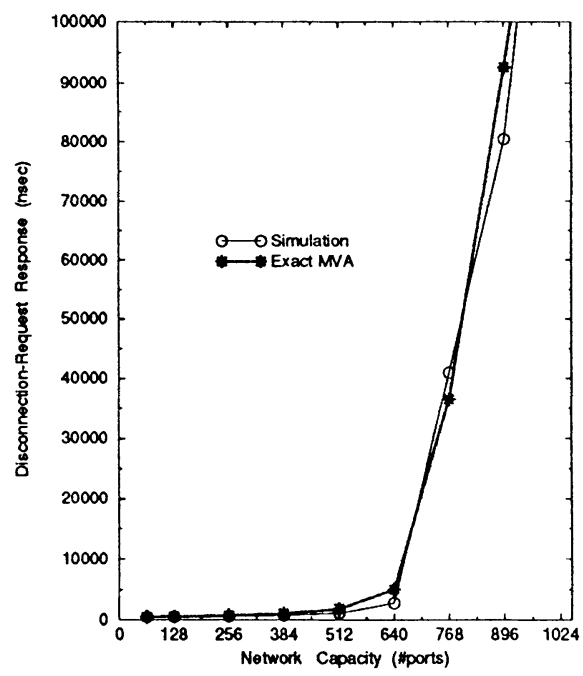

(b)

Figure 18. Router Latency for Disconnection-Requests 


\section{CONCLUDING REMARKS}

In this paper, we considered the nonblocking operation of asymmetrical three-stage Clos networks. We first considered a control algorithm developed by Ohta [17] for symmetric networks. We derived general conditions under which the asymmetrical network is nonblocking under Ohta's control algorithm.

Ohta's algorithm (which is referred to as ALGO1) operates by attempting to balance the number of established connections between any pair of input and output switches among all middle-stage switches. This scheme does not work well when faulty links exist in the network, since it does not keep track of the available paths between input and output switches. Due to faults, some paths are no longer available and certain decisions of ALGO1 result in increased blocking probability.

To achieve better performance for asymmetrical networks, we designed a new algorithm (ALGO2), which attempts to balance the number of remaining available paths between any pair of input and output switches among all middle-stage switches. Both algorithms were tested with random sequences of requests, which included connections and disconnections. Simulation results show that ALGO2 outperforms ALGO1 in most cases for a variety of fault patterns and load conditions. In addition, these results indicate that ALGO2 is more likely to achieve higher network utilization and smaller blocking probability, especially in networks with faults.

To complete the evaluation of our semi-centralized algorithm, we subsequently considered circuit switching networks with semi-centralized routers. We developed a simple queuing model for such a router, and used an exact mean value analysis technique to solve it. The algorithm that we used has linear execution time with respect to the capacity of the network and is very efficient.

We validated our model, by comparing its predictions with results from an event-driven simulator of the modeled system, and we demonstrated its accuracy for throughput and capacity utilization predictions. The error for those measures is around $0.5 \%$ for most of the cases we tested. However, response times for connection and disconnection requests are not predicted accurately, especially as the router begins to saturate. One reason for this error is due to the fact that, in saturated conditions, the router becomes a strong bottleneck and the entire system cannot converge to the steady state very fast. Thus, the simulator results are "polluted" due to a long start-up effect (or the cold-start period), and do not reflect the steady state predicted by the model. Moreover, strong bottlenecks tend to converge to a steady state very slowly. Therefore, one requires long simulation runs to evaluate real and complex switching networks.

The contribution of our method is a very fast and efficient way to have a relatively accurate estimate of the performance of a switching network. Fast performance evaluations are necessary for making efficient switch-design choices, as well as for fine-tuning the performance of existing networks.

For low network capacities, relatively fast centralized routers may perform adequately. When the router saturates, its response time increases very fast and may eventually become a strong bottleneck, resulting in the network under-utilization. Such a problem may be alleviated by exploiting all the available parallelism that exists in the decisionmaking process of the router; this, in turn, requires semi-distributed, parallel router 
designs.

Knowing the latency of a connection or disconnection request, we can design our data buffers accordingly and we can overlap data-transmission and the router's decision-making in such a way that the main data stream incurs the least possible delay due to the routing overhead.

We are currently investigating a few other control algorithms for nonblocking operation of asymmetrical Clos networks. In the future, we plan to evaluate these algorithms with respect to different measures such as network utilization, blocking probability and faulttolerance. In addition, we are trying to improve the accuracy of our model, and to expand its range of applications. At the same time we use it as a tool to evaluate the performance of our router designs.

\section{Acknowledgements}

The authors would like to thank Prof. Anujan Varma of the University of California at Santa Cruz for his valuable comments during the course of this work.

\section{REFERENCES}

[1] J. Beetem, M. Denneau, and D. Weingarten, "The GF11 supercomputer," Proc. of the 12th Annual Int. Symposium on Computer Architecture, pp.108-115, June 1985.

[2] V.E. Beneš, Mathematical Theory of Connecting Networks and Telephone Traffic, Academic Press, New York, 1965.

[3] J.-Y. Le Boudec, "The Asynchronous Transfer Mode: a tutorial", Computer Networks and ISDN Systems, 24, pp.279-309, 1992.

[4] C. Clos, "A study of non-blocking switching networks," Bell Syst. Tech.J., pp.406-424, March 1953.

[5] P. Coppo, M. D'Ambrosio, and R. Melen, "Optimal Cost/Performance Design of ATM Switches," IEEE Transactions on Networking, Vol. 1, No. 5, Oct. 1993, pp. 566-575.

[6] B. G. Douglass, "A new class of three-stage switching networks and their routing properties," Proceedings of the Int. Conference on Parallel Processing, pp.397-400, 1990.

[7] F. K. Hwang, "Rearrangeability of multiconnection three-stage Clos networks," Networks 2, pp.301-306, 1972.

[8] F. K. Hwang, "Control algorithms for rearrangeable Clos networks," IEEE Trans. Commun. COM-31(8), pp.952-954, 1983.

[9] F. K. Hwang and A. Jajszczyk, "On nonblocking multiconnection networks," IEEE Trans. Commun. COM-34, pp.1038-1041, 1986.

[10] G. C. Hudek, "Blocking in digital cross-connect systems," IEEE Network, pp.34-39, Jan. 1993.

[11]R. Jain, The Art of Computer Systems Performance Analysis, ch.34, pp.575-584, J.Wiley \& Sons, New York, 1991.

[12] A. Jajszczyk, "On nonblocking switching networks composed of digital symmetrical matrices", IEEE Trans. Commun. COM-31, pp.2-9, 1983.

[13] D. M. Koppelman, "A self-routing permutation network, a classification of all three- 
stage networks, some other permutation network designs and performance bounds," Ph.D. Thesis, Rensselaer Polytechnic Institute, 1988.

[14]E. D. Lazowska, J. Zahorjan, G. S. Graham, K. C. Sevcik, Quantitative System Performance, ch.4,6,7, Prentice Hall, N.J. 1984.

[15]F. K. Liotopoulos and S. Chalasani, "Nonblocking Operation of Asymmetrical Clos Networks," Proc. 23rd Int. Conf. of Parallel Processing, vol.I, pp.101-108, Chicago, USA, August 1994.

[16] G. M. Masson and B. W. Jordan, "Realization of multiple.connection assignment with asymmetrical multistage connection networks," Networks 2(3), 1972.

[17]S. Ohta, "A Simple Control Algorithm for Rearrangeable Switching Networks with Time Division Multiplexed Links," IEEE Journal on Sel. Areas in Comm., vol.SAC-5, no.8, pp.1302-1308, Oct.1987.

[18] M.C. Paull, "Reswitching of Connection Networks," Bell Syst. Tech. J., Vol. 41, pp.833-855, 1962.

[19]B. Saleh, M. C. Teich, "Photonic Switching and Computing", Fundamentals of Photonics, ch.21, pp.832-873, J.Wiley \& Sons, New York, 1991.

[20] W. Stallings, Local Networks, ch.7, pp.240-280, Macmillan Publishing Company, 3rd edition, New York, 1990.

[21]A. Varma and S. Chalasani, "Asymmetrical Multiconnection Three-Stage Clos Networks," Networks, vol.23 (1993), pp.427-439.

[22] A. Varma, V. Sahai, and R. Bryant, "Performance Evaluation of a High-Speed Switching System Based on the Fibre Channel Standard," Proceedings of the Second International Symposium on High Performance Distributed Computing, 1993, pp. 144-151.

\section{APPENDIX A}

To prove Lemma 1, we need the following lemma.

Lemma 0: In the network for which ALGO1 is applied:

$C(i, j, k) \leq \min _{p} C(i, p, k)+1, \quad \forall i, j, k$

Proof of Lemma 0: (by induction on the number of requests) Initially, all $C(i, j, k)=0$ and (4) is satisfied. Assume that (4) is satisfied after a certain number of connections and disconnections.

Let a connection request from $I$ to $K$, be routed by ALGO1 through the middle-stage switch $J$. Then, only $C(I, J, K)$ is increased by one. The minimum is not affected, and, by the selection criterion, $C(I, J, K)$ will now become equal to $\min _{p} C(I, p, K)+1$. Therefore, (4) is satisfied even after this connection.

Assume a disconnection request for $[I, J, K]$. If a rearrangement is necessary, then let $\left[I, J_{m}, K\right]$ be rearranged as $[I, J, K]$ as per the disconnection rule. Let $J_{s}=J_{m}$, if a rearrangement is performed and $J_{s}=J$, otherwise. In both cases, according to the definition of the algorithm, $C\left(I, J_{s}, K\right)=\max _{p} C(I, p, K)$ will be the only one to be decreased by one. But then, $C\left(I, J_{s}, K\right)=\min _{p} C(I, p, K)$, and (4) still holds.

Proof of Lemma 1: (Sufficiency) Suppose that a connection from first-stage switch $I$ to third-stage switch $K$ is established through middle-stage switch $J$. Before the connection, 
let $A_{I, j}$ be the number of links used between first-stage switch $I$ and middle-stage switch $j$. Clearly,

$$
A_{I, j}=\sum_{k=1}^{g} C(I, j, k)
$$

Since the algorithm selects the middle-stage switch $J$,

$C(I, J, K)=\min _{p} C(I, p, K)$

From Lemma 0,

$C(I, J, k) \leq \min _{p} C(I, p, k)+1, \quad(k \neq K)$

From (5),(6), and (7),

$A_{I, J} \leq \sum_{k=1}^{g} \min _{p} C(I, p, k)+g-1$

Clearly, from (5),

$\sum_{k=1}^{g} \min _{p} C(I, p, k) \leq A_{I, j}$

From (8) and (9),

$A_{I, J}-g+1 \leq A_{I, j}$

Because the new connection is feasible, there is at least one idle link originating from first-stage switch $I$. Therefore, the total number of used links cannot be greater than $P_{I}-1$. Or,

$A_{I, J}+\sum_{\substack{j=1 \\ j \neq J}}^{m} A_{I, j} \leq P_{I}-1$

From (10) and (11),

$m \cdot A_{I, J} \leq P_{I}-g+m \cdot(g-1)$

and noting that $A_{I, J}$ is an integer,

$A_{I, J} \leq\left\lfloor\frac{P_{I}-g}{m}\right\rfloor+g-1$

In a similar way, (duality principle), the number of links used between the middle-stage switch $J$ and the middle-stage switch $K, B_{J, K}$, is:

$B_{J, K} \leq\left\lfloor\frac{Q_{K}-f}{m}\right\rfloor+f-1$

Consequently, a new path can always be established, if the number of links $R_{I, J}$ and $T_{J, K}$ are at least one more than the quantities on the right sides of (13) and (14). Thus, if (2) and (3) are satisfied, the connection from $I$ to $K$ can be established. 